\title{
Recommendation of RILEM TC237-SIB on fragmentation test for recycled asphalt
}

\author{
Gabriele Tebaldi (1) Eshan Dave $\cdot$ Augusto Cannone Falchetto $\cdot$ \\ Martin Hugener • Daniel Perraton - Andrea Grilli • Davide Lo Presti • \\ Marco Pasetto • Andreas Loizos • Kim Jenkins • Alex Apeagyei • \\ James Grenfell · Maurizio Bocci
}

Received: 22 January 2019/Accepted: 30 May 2019/Published online: 25 July 2019

(C) RILEM 2019

\begin{abstract}
This recommendation describes how to characterize the reclaimed asphalt through the fragmentation test. The guidelines given hereafter are based on the results of a round robin test organized by the RILEM Technical Committee 237-SIB “Testing and characterization of sustainable innovative bituminous materials and systems"' and provide information on the testing procedure, data analysis and indications for the preparation of a test report.
\end{abstract}

This recommendation was developed by the task group TG6 within RILEM TC 237-SIB consisting of Gabriele Tebaldi, Eshan Dave, Martin Hugener, Augusto Cannone Falchetto, Daniel Perraton, Andrea Grilli, Davide Lo Presti, Marco Pasetto, Andreas Loizos, Kim Jenkins, Alex Apeagyei, James Grenfell, Maurizio Bocci. The draft recommendation was submitted for approval to the full TC and subsequently approved by RILEM TC 237-SIB.

TC Chairman: Manfred N. Partl (EMPA—Swiss Federal Laboratories for Materials Science and Technology, Dubendorf, Switzerland).

TC Deputy Chair: Emmanuel Chailleux (IFSTTAR-French institute of science and technology for transport, development and networks, Nantes, France).

TG6 Group Leader: Gabriele Tebaldi (University of Parma, Italy_University of Florida, USA).

TC Members: Gordon Airey (UK), Alex Apeagyei (UK), Ignacio Artamendi (UK), Hassan Baaj (Canada), Hussain U. Bahia (USA), Wojciech Bankowski (Poland), Davide Broere (The Netherlands), Maurizio Bocci (Italy), Yves Brosseaud (France), Willam G.
Keywords Reclaimed asphalt (RA) $\cdot$ Round robin test (RRT) · Fragmentation test

\section{Introduction}

The use of reclaimed asphalt (RA) in asphalt mixture is common practice in the pavement construction industry, due to both economical and environmental benefits

Buttlar (USA), Francesco Canestrari (Italy), Armelle Chabot (France), Emmanuel Chailleux (France), Eshan Dave (USA), Joëlle De Visscher, (Belgium), Hervé Di Benedetto (France), Augusto Cannone Falchetto (Germany), Gilda Ferrotti (Italy), Tomas Gabet (France), Marcin Gajewski (Poland), Vincent Gaudefroy (France), William Grady (The Netherlands), Andrea Graziani (Italy), James Grenfell (UK), Andrea Grilli (San Marino), Ferhat Hammoum (France), Bernhard Hofko (Austria), Martin Hugener(Switzerland), Illan Ishai (Israel), Kim Jenkins (South Africa), Nicole Kringos (Sweden), Greet A.Leegwater (The Netherlands), Eyal Levenberg (Israel), Andreas Loizos (Greece), Davide Lo Presti (UK), Xiaohu Lu (Sweden), Paul Marsac (France), Anne Millien (France), Konrad Mollenhauer (Germany), Francisco Morea (Argentina), Virginie Mouillet (France), Patrick Muraya (Norway), Marco Pasetto (Italy), Manfred N. Partl(Switzerland), Daniel Perraton (Canada), Christophe Petit (France), Katherine Petros (USA), Laurent Porot (The Netherlands), Simon Pouget (France), Lily D. Poulikakos (Switzerland), Christiane Raab (Switzerland), Safwat Said (Sweden), Cesare Sangiorgi (Italy), Cédric Sauzéat (France), Tom Scarpas (The Netherlands), Hilde Soenen (Belgium), Dariusz Sybilsky (Poland), Gabriele Tebaldi (Italy), Ann Vanelstraete (Belgium), Stefan Vansteenkiste (Belgium), Michael Wistuba (Germany), Ludo Zanzotto (Canada), Adam Zofka (Poland). 
[1]. This material is obtained from old asphalt pavement at the end of their service life after a milling or a demolition process. RA mainly consists of aggregates, aged and oxidized bitumen and mastic and, in many instances, it appears as a conglomerate of multiple aggregate particles of different sizes and shapes.

The particle size distribution of RA depends on a number of factors: (1) the original asphalt material; (2) the specific milling and demolition devices, operations and conditions; (3) the extent of distress conditions and the current aging; and (4) the handling of the milled RA. In addition, during the construction process, the particle size distribution of RA evolves due to mechanical actions (mixing, handling, compaction etc.) and temperature variations (during storage and mixing). Therefore, it is necessary to know this evolution to implement a reliable mix design process since the mechanical performance of the recycled asphalt mixture is heavily dependent on the gradation curve of RA. For example, previous studies [2-6] have shown that even small changes in the grading curve can have a significant impact on the mixture performance, and as a result, it is extremely important to keep the aggregate sizes and their distribution under control. Moreover, the evolution of the particle size distribution of RA under mechanical actions is specific to each RA source, prompting the need of including this change in

G. Tebaldi ( $₫)$

University of Parma, Parma, Italy

e-mail: gtebaldi@unipr.it

G. Tebaldi

University of Florida, Gainesville, USA

E. Dave

Civil and Environmental Engineering, University of New Hampshire, Durham, USA

A. Cannone Falchetto

Technische Universitat Braunschweig, Braunschweig, Germany

M. Hugener

EMPA, Swiss Federal Laboratories for Materials Science and Technology, Dübendorf, Switzerland

D. Perraton

University of Québec, ETS, Montreal, Canada

A. Grilli

University of San Marino, San Marino, San Marino gradation into a "fingerprinting" protocol for characterizing RA material.

Because RA is extremely heterogeneous, this is not a simple challenge, particularly during the quality control phases. The main difficulty lies in the fact that the conventional methods used to classify RA do not involve a quick and simple test that can be used in the laboratory to produce reference values and to secure fast data that may be compared with those references values in the field operation. In addition, there is no other test procedure to characterize the evolution of RA particle size distribution at present.

\section{Scope}

The present recommendation gives guidance for a new experimental procedure to differentiate the RA from different sources. Specifically, it refers to the test procedure of the fragmentation test. This recommendation is based on the results of a round robin test (RRT) organized by the RILEM Technical Committee 237-SIB TG 6 "Testing and characterization of sustainable innovative bituminous materials and systems-Cold Recycling". This document contains guidelines on material preparation, testing procedure, data analysis and presentation of results. The proposed testing method fill a gap in existing international

D. Lo Presti

University of Nottingham, Nottingham, UK

M. Pasetto

University of Padova, Padova, Italy

A. Loizos

National Technical University of Athens, Athens, Greece

K. Jenkins

Stellenbosch University, Stellenbosch, South Africa

A. Apeagyei

University of East London, London, UK

J. Grenfell

Australian Road Research Board, Port Melbourne,

Australia

M. Bocci

Università Politecnica delle Marche, Ancona, Italy 
standards; additional details on the results of the RRT can be found in Tebaldi et al. [7-9].

The application of this test procedure is intended as part of an RA characterization protocol as well as for use in mix design procedures of asphalt mixtures that incorporate RA. It is recommended that this test is used to enhance the reliability of mix design procedures so that the final product in field operation is comparable to the designs conducted in the laboratory. In addition, since the recommended procedure includes testing at different temperatures, results provide insight on the availability of bitumen from RA, which can be potentially reactivated, as a function of temperature and characterize the influence of temperature on the evolution of particle size distribution. Furthermore, the test method can provide a first guidance in decisions regarding the selection of the specific recycling technology: hot, warm or cold recycling.

\section{Referenced documents}

The following section provides a list of international standards and documents linked to the present recommendation.

\subsection{ASTM standards}

- ASTM C136/C136 M-14 (2014) Standard Test Method for Sieve Analysis of Fine and Coarse Aggregates, ASTM International, West Conshohocken, PA.

- ASTM C702/C702 M-11 (2011) Standard Practice for Reducing Samples of Aggregate to Testing Size, ASTM International, West Conshohocken, PA, 2011.

- ASTM D1557-12 2012) Standard Test Methods for Laboratory Compaction Characteristics of Soil Using Modified Effort (56,000 ft-lbf/ $\mathrm{ft}^{3}(2700 \mathrm{kN}-$ $\left.\mathrm{m} / \mathrm{m}^{3}\right)$ ), ASTM International, West Conshohocken, PA.

\subsection{EN standards}

- EN 933-1 (2012) Tests for geometrical properties of aggregates-Part 1: Determination of particle size
distribution-Sieving method. European Committee for Standardization, Brussels, Belgium.

- EN 13108-8 (2013) Bituminous mixtures-Material specifications-Part 8: Reclaimed asphalt. European Committee for Standardization, Brussels, Belgium.

- EN 13286-2 (2012) Unbound and hydraulically bound mixtures-Part 2: Test methods for laboratory reference density and water content-Proctor compaction. European Committee for Standardization, Brussels, Belgium [10-15].

\section{Definitions}

Please refer to "Recommendation of RILEM TC237SIB: Protocol for Characterization of Recycled Asphalt (RA) Materials for Pavement Applications" for definitions that are relevant to this recommendation.

\section{Materials preparation}

The RA to be tested can be obtained from different sources, different asphalt mixtures, prepared for different pavement layers. It can differ in asphalt binder type and content, aggregate type, particles size and distribution, milling operation pavement conditions and distresses, storage time and conditions (protected or unprotected stockpiles).

The initial sample amount has to be large enough to be representative for the RA origin and sufficient to complete the fragmentation test. For the pre-treated RA samples, no specific protocol is recommended. Nevertheless, it may be advisable to air dry the material at room temperature for $24 \mathrm{~h}$ and then place it in a thermostatically controlled oven at a temperature of $40{ }^{\circ} \mathrm{C}$ for $24 \mathrm{~h}$ prior to testing. Homogenization of the main samples before reducing them to test samples is standard practice.

\section{Test procedure}

The fragmentation test provides an indication on the particle resistance to fragmentation under a series of shocks induced by dropping a steel mass, namely a 
hammer, on a confined sample placed in a steel cylindrical mould.

The fragmentation test measures the amount of RA passing through a control sieve after a fixed series of strokes carried out with a normalized falling mass. The material crushed to sizes finer than a specified control sieve is separated and expressed as a percentage of the initial weight of the material placed in the mould. The passing through the control sieve (PCS) is expressed as percentage of the initial weight of the material and recorded.

The test is performed on different aggregate fractions $20 / 30 \mathrm{~mm}, 14 / 20 \mathrm{~mm}, 10 / 14 \mathrm{~mm}$, and $5 / 10 \mathrm{~mm}$. The first number of the fraction represents the size of the sieve for which $100 \%$ of material is retained, while the second number refers to the size of the sieve where $100 \%$ of material is passing. Figure 1 shows a schematic of the fragmentation test procedure: the material is first sieved (Fig. 1a, b) and then compacted in five layers (Fig. 1c) with 56 number of blows per layer using a standardized falling mass. Finally, the fine material produced by the impact of the hammer is sieved with a control sieve (PCS) (Fig. 2d). The test is repeated at three different temperatures: 5, 20 and $40{ }^{\circ} \mathrm{C}$, to evaluate the difference in material response under different conditions.

\section{Data analysis}

Since the grading curve influences the mixture performance, it is extremely important to keep under control the aggregate sizes and their distribution. The aim of the fragmentation test is to predict the reliability of the gradation of a granular mix taking in consideration the accidental changes caused by the breaking of RA conglomerates. As mentioned before, four fraction classes are considered: 20/30 mm, 14/ $20 \mathrm{~mm}, 10 / 14 \mathrm{~mm}$ and 5/10 mm. For each fraction, RA particles are completely retained on the smaller size sieve and pass through the larger size one, while the mean particle size between two consecutive sieves is defined as:

Mean particle size

$$
=\sqrt{\text { size of the sieve ' } i \text { ' } \times \text { size } \text { of the sieve ' } i+1} \text { ' }
$$

The analysis of the data should include the evolution of the material passing through the control sieve (PCS) produced under fragmentation as a function of temperature.

The coefficient of variation: $\mathrm{CV}=$ (standard deviation/mean value) $\times 100$, can be used to calculate the dispersion of the results in terms of mean particle size for the different testing temperatures.

\subsection{Categorization of RA based on fragmentation potential}

To classify RA with respect to its susceptibility to undergo size changes while in use, the fragmentation results obtained at $5{ }^{\circ} \mathrm{C}$ can be compared to those derived from fragmentation tests performed on virgin aggregate, according to the categories defined in the EN 12620 [16] standard and presented in Table 1.
Fig. 1 Procedure and apparatus used in the fragmentation tests (ASTM D 1557, 2012)

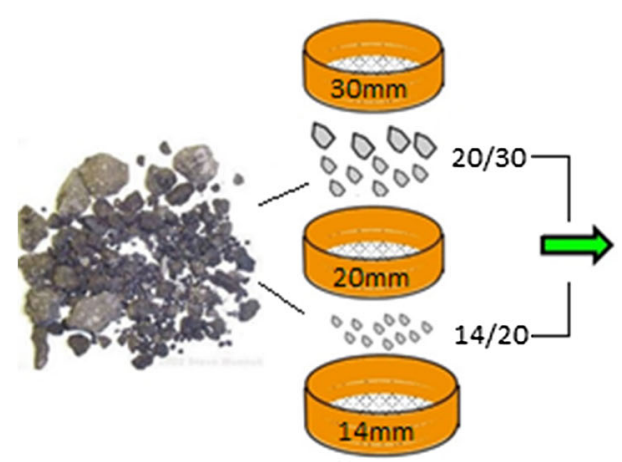

$\mathbf{a}$

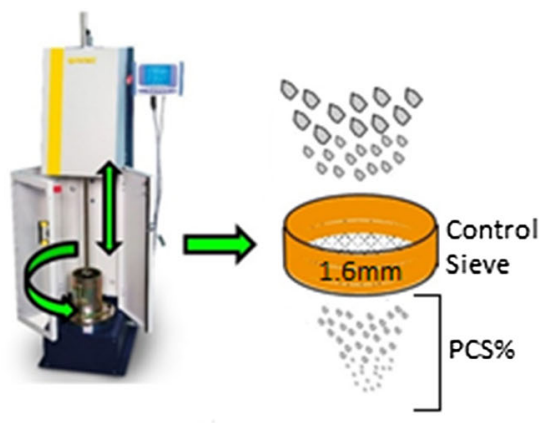

c 
Fig. 2 Schematic representation of the relationship between the percent passing through the control sieve (PSC (\%)) and the testing temperature

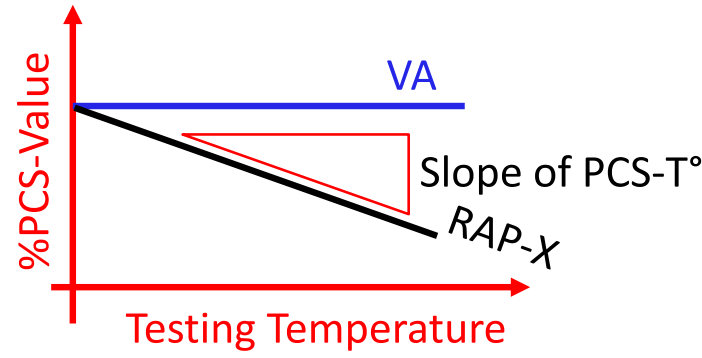

Slope $=\frac{P C S_{T=5^{\circ} C}-P C S_{T=x^{\circ} C}}{\Delta T}$
Table 1 RA categories and limits with respect to the vulnerability of RAP particles to change size while in use and the corresponding classification of RAPs tested

\begin{tabular}{|c|c|c|c|c|}
\hline Categories & B & $\mathrm{C}$ & D & $\mathrm{E}$ \\
\hline Limits on $\mathrm{PCS}_{5}{ }^{\circ} \mathrm{C}_{-} 5 / 10 \mathrm{~mm}(\%)$ & $\leq 7$ & $\leq 9$ & $\leq 11$ & $\leq 14$ \\
\hline
\end{tabular}

\subsection{Categorization of RA based on agglomerate potential}

The fragmentation test results obtained at different temperatures provide the possibility of verifying the agglomerate potential of a RA material. The impact of RA-source on the agglomeration potential of a RA can be linked to the slope of the $\mathrm{PCS}-\mathrm{T}^{\circ}$ relationship as exhibited in Fig. 2.

To compare the slopes of the PSC-T relationship from all data, RA source results for each specific fraction group, need to be first normalized with respect to the PCS result obtained at $5{ }^{\circ} \mathrm{C}\left(\mathrm{PCS}_{\mathrm{STD}}\right.$ at $\left.5{ }^{\circ} \mathrm{C}\right)$ as defined in equation (Eq. 2). A standardized value of 1 means that the temperature does not affect the fragmentation results.

$\operatorname{PCS}_{\mathrm{STD} a t 5^{\circ} \mathrm{C}}=\frac{\mathrm{PCS}_{T^{\circ} C}(\%)}{\mathrm{PCS}_{T=5^{\circ} C}(\%)}$

By assuming a linear relationship, the thermal susceptibility can be linked to the slope value of the $\mathrm{PCS}_{\mathrm{STD}}$ at $5{ }^{\circ} \mathrm{C}-\mathrm{vs} \mathrm{T}^{\circ}$ relationship and this can be used as criterion for define categories and limits to classify the RA as shown in Table 2.
Table 2 RA categories and limits with respect to the vulnerability of RAP particles to change size in its use and the corresponding classification of RAP tested

\begin{tabular}{lllll}
\hline Categories & 1 & 2 & 3 & 4 \\
\hline $\begin{array}{l}\text { Absolute value of the rate of } \\
\text { change of } \mathrm{PCS}\end{array}$ & $\leq 5$ & $\leq 10$ & $\leq 18$ & $\leq 25$ \\
temperature $\left(\times 10^{3}\right)\left({ }^{\circ}{ }^{\circ} \mathrm{C}\right.$ with &
\end{tabular}

\section{Closing remark and test report}

The fragmentation test can be used to characterize RA and possibly to classify it according to the source. Based on the present document the test report should contain

- Relevant information on the tested RA material, such as origin, storing, and potential conditioning;

- The description of the test setup, including: mould size, rammer weight, height of fall, blows per layer, number of layers, control sieve;

- The values measured for each size class, and temperature, the weight of each class before and after hammering and the coefficient of fragmentation $(\mathrm{PCS} \%)$;

- The value of the mean particle size and coefficient of variation;

- The category of RA in terms of fragmentation potential and agglomerate potential.

Author contribution's Elie Hajj, Patrick Muraya, Giovanni Giacomello, Di Wang have contibuted equally to this work.

\section{Compliance with ethical standards}

Conflict of interest The authors declare that they have no conflict of interest. 


\section{References}

1. Kennedy TW, Tam WO, Solaimanian M (1998) Optimizing use of reclaimed asphalt pavement with the Superpave system. J Assoc Asph Paving Technol 67:311-325

2. Mansour TN, Putman BJ (2012) Influence of aggregate gradation on the performance properties of porous asphalt mixtures. J Mater Civil Eng 25(2):281-288. https://doi.org/ 10.1061/(ASCE)MT.1943-5533.0000602

3. Isola M, Zou J, Roque R, Lopp G (2014) Evaluation of gradation-based criteria for cracking performance. Asphalt Pavements-Kim(Ed). Taylor \& Francis Group, London

4. Isola M (2014) Effect of interstitial volume characteristics on asphalt mixture durability and cracking performance. $\mathrm{PhD}$ thesis, University of Florida

5. Afaf AHM (2014) Effect of aggregate gradation and type on hot asphalt concrete mix properties. J Eng Sci Assiut Univ 42(3):567-574

6. Al-Mosawe H, Thom N, Airey G, Al-Bayati A (2015) Effect of aggregate gradation on the stiffness of asphalt mixtures. J Pavement Eng Asph Technol 16(2):39-49. https://doi.org/ 10.1515/ijpeat-2015-0008

7. Tebaldi G, Dave E, Marsac P, Muraya P, Hugener M, Pasetto $M$ et al (2012a) Classification of recycled asphalt (RA) material. In: 2nd International symposium on asphalt pavements \& environment, ISAP TC APE, Fortaleza. Brazil

8. Tebaldi G, Dave EV, Marsac P, Muraya P, Hugener M, Pasetto M et al (2012b) Synthesis of specimen preparation and curing processes for cold recycled Asphalt mixes. In: 2nd International symposium on asphalt pavements \& environment, ISAP TC APE, Fortaleza. Brazil
9. Tebaldi G, Dave EV, Marsac P, Muraya P, Hugener M, Pasetto M et al (2014) Synthesis of standards and procedures for specimen preparation and in-field evaluation of cold recycled asphalt mixtures. Road Mater Pavement 15(2):272-299. https://doi.org/10.1080/14680629.2013. 866707

10. McGarrah EJ (2007) Evaluation of current practices of reclaimed asphalt pavement/virgin aggregate as base course material (No. WA-RD 713.1)

11. EN 12697-42 (2012) Bituminous mixtures. Test methods for hot mix asphalt. Amount of foreign matter in reclaimed asphalt. European Committee for Standardization, Brussels

12. EN 13108-8 (2013) Bituminous mixtures-material specifications-part 8: reclaimed asphalt. European Committee for Standardization, Brussels

13. Recycling Cold (2010) Wirtgen cold recycling technology, 3rd edn. Wirtgen $\mathrm{GmBH}$, Windhagen

14. EN 13286-2 (2012) Unbound and hydraulically bound mixtures-part 2: test methods for laboratory reference density and water content-proctor compaction. European Committee for Standardization, Brussels

15. ASTM D1557-12 (2012) Standard test methods for laboratory compaction characteristics of soil using modified effort $\left(56,000 \mathrm{ft}-\mathrm{lbf} / \mathrm{ft}^{3}\left(2700 \mathrm{kN}-\mathrm{m} / \mathrm{m}^{3}\right)\right)$. ASTM International, West Conshohocken

16. EN 12620 (2013) Aggregates for concrete. European Committee for Standardization, Brussels

Publisher's Note Springer Nature remains neutral with regard to jurisdictional claims in published maps and institutional affiliations. 